\title{
Identification of Polyomaviruses in Skin Cancers
}

\author{
Pedro V.A. Costa ${ }^{a}$ Patricia S. Ishiy ${ }^{a}$ Paulo R.P. Urbano ${ }^{b}$ Camila M. Romano $^{b}$ \\ Stephen K. Tyring ${ }^{c}$ Walmar R.P. Oliveira ${ }^{a}$ Cyro Festa-Neto ${ }^{a}$ \\ aDepartment of Dermatology, Clinics Hospital of the Medical School of the University of São Paulo, São Paulo, Brazil; \\ bVirology Research Laboratory (LIMHC 52), Clinics Hospital of the Medical School of the University of São Paulo, São \\ Paulo, Brazil; 'Department of Dermatology, University of Texas Health Science Center, Houston, TX, USA
}

\section{Keywords}

Polyomavirus · Skin neoplasms · Carcinogenesis

\section{Abstract}

Background: Polyomaviruses (PyVs) were initially described in animals. They have also been detected in humans with some evidence that could play a role in skin carcinogenesis. Objectives: This study aimed to verify the presence of PyVs in different skin tumour samples and to make clinical correlations with patients' epidemiological data from Clinics Hospital of Medical School of University of São Paulo, Brazil. Methods: This is a cross-sectional study. A random selection was performed of 120 patients with histopathological exams of different cutaneous neoplasms equally divided into 6 groups and 20 patients with normal skin. The available skin specimens were analysed with 2 different techniques of PCR (conventional and real time) for detection of PyV DNA. Concomitantly, retrospective analysis of the respective medical records for the collection of epidemiological data was done. Analyses suitable for categorical data were used to compare the proportion of patients in each group. Results: PyV DNA was found in $25.69 \%$ of the samples: $15 \%$ in basal cell carcinoma group, $15 \%$ in squamous cell carcinoma, $28.57 \%$ in melanoma, $15 \%$ in dermatofibrosarcoma protuberans, $13.33 \%$ in Kaposi sarcoma, $65 \%$ in Merkel cell carcinoma (MCC), and none in normal skin. Merkel cell PyV detection was statistically significant in MCC patients ( $p$ value $<0.01$ ), but no correlations were found between PyVs and others skin tumours. Conclusion: This study demonstrated the presence of PyVs in different skin tumours; however, no association of any PyVs found in any skin tumour with epidemiological data could be shown. Further studies are still needed to elucidate the mechanisms of PyVs in skin carcinogenesis.

(C) 2021 S. Karger AG, Basel

\section{Introduction}

Polyomaviruses (PyVs) are small, non-enveloped viruses with a circular double-stranded DNA genome of approximately 5,000 base pairs, surrounded by an icosahedral capsid composed of 3 structural proteins VP1, karger@karger.com www.karger.com/int

(C) 2021 S. Karger AG, Basel

Karger
Walmar R.P. Oliveira

Department of Dermatology

Clinics Hospital of the Medical School of the University of São Paulo

Doutor Enéas de Carvalho Aguia, São Paulo 05403-000 (Brazil)

walmar.roncalli@hc.fm.usp.br 
VP2, and VP3. Although PyVs were initially described in mice, they have been continuously detected in different bird species, cattle, bats, rabbits, hamsters, sea lions, and monkeys, as well as in humans. A classic example of a non-human $\mathrm{PyV}$ is the rhesus monkey $\mathrm{PyV}$ simian virus 40 (SV40) [1].

The first human PyVs (HPyVs) described were BK virus (BKPyV) and JC virus (JCPyV), associated, respectively, with nephropathy and progressive multifocal leukoencephalopathy in immunosuppressed patients. Since then, 12 other $\mathrm{HPyV}$ species have been identified, of which 9 have been detected in the skin: Merkel cell PyV (MCPyV), Trichodysplasia spinulosa-associated PyV (TSPyV), HPyV6, HPyV7, HPyV9, HPyV10 (MWPyV or MXPyV), HPyV13, Saint Louis PyV (STLPyV), and, more recently, Lyon IARC PyV (LIPyV). Only MCPyV, TSPyV, HPyV6, and HPyV7 were definitely related to skin diseases, predominantly in immunocompromised patients [2-4].

The identification of $\mathrm{MCPyV}$ as an etiologic agent of Merkel cell carcinoma (MCC) was the first evidence that an $\mathrm{HPyV}$ could play an important role in skin carcinogenesis. MCPyV DNA is present in approximately $80 \%$ of MCC. The integration of viral DNA into the host genome, as well as its potential to drive the expression of some viral oncoproteins in host cells, reinforces its pivotal role in oncogenesis $[5,6]$. MCPyV has been found in other tumours, particularly skin cancers, such as basal cell carcinoma (BCC), squamous cell carcinoma (SCC), keratoacanthoma, and melanoma $[1,2]$.

HPyVs encode the oncoproteins and large and small T-antigens. However, the oncogenic potential has been established only for MCPyV in MCC development. The most important mechanism of malignant transformation associated with PyVs is the genetic or functional uncoupling of T-antigen expression from the later steps of the viral life cycle consisting of viral DNA replication, late gene expression, virion assembly, and eventually, host cell lysis. Consequent to this uncoupling, cells are left with the T-antigens subverting cell cycle control by inactivating signal transduction pathways and tumour suppressor proteins, $\mathrm{pRB}$ and $\mathrm{p} 53$, in route to neoplastic transformation [1, 5, 7-9].

This study aims to verify the presence of HPyVs in different skin tumour samples in paraffin waxed material from the Dermatopathology Laboratory of the Dermatology Division of Clinics Hospital of Medical School at the University of São Paulo, through the viral DNA detection by PCR. It also seeks to investigate the possible correlation of $\mathrm{HPyV}$ DNA present in different skin tumours such as BCC, SCC, melanoma, dermatofibrosarcoma protuberans (DFSP), Kaposi sarcoma, and MCC with patients' epidemiological data.

\section{Materials and Methods}

This is a cross-sectional study, conducted at the Clinics Hospital of the Medical School of the University of São Paulo, Brazil. There was a random selection of 120 patients with histopathological exams of different cutaneous neoplasms, equally divided into 6 groups according to the diagnostic results, totalling 20 patients in each group. In addition, there was a group of 20 random samples of normal skin biopsies. The histopathological exams were cutaneous tumours in formalin-fixed and paraffin-embedded material (FFPEM) in $6 \mathrm{~mm}$ sections, filed in the Dermatopathology Laboratory from 1994 to 2018. The selection of the study size was made according to the skin samples availability, as well as material for PyV DNA analysis. All samples were retrieved from the archives and had been excised for diagnostic and/or therapeutic reasons of patients aged 18 or over. The patients were divided into: group 1 - BCC; group 2 - SCC; group 3 - melanoma; group 4 - DFSP; group 5 - KS; group 6 - MCC, and group 7 - normal skin.

Concomitantly, a retrospective analysis of the respective medical records was made for the collection of epidemiological data such as sex, age, colour, site of the tumour, personal history of skin cancer, and state of immunosuppression, aiming to establish any correlation of these data with the presence of HPyV infection. The missing data were not included in the statistics and results.

Continuous variables were expressed in terms of summary measures (mean, median, and standard deviation), while categorical variables were expressed in terms of percentages. Bar charts were used to illustrate categorical variables, while boxplots were used to illustrate the continuous variables. Fisher's exact test was used to measure the associations between tumour types and immunosuppression versus $\mathrm{PyV}$. The $\mathrm{R}$ version 3.5.0 software was used to carry out all analyses.

\section{Analysis of PyV DNA}

The extraction of viral DNA from FFPEM with the NucleoSpin ${ }^{\circledR}$ Tissue kit was made according to the manufacturer's instructions (Macherey-Nagel ${ }^{\circledR}$ ). The amount of DNA ranged from $1.0 \mathrm{ng} / \mathrm{uL}$ to $150 \mathrm{ng} / \mathrm{uL}$ for quantification of the samples and test for an endogenous GAPDH gene confirmed human DNA after extraction. Moreover, in order to check if FFPEM DNA had been recovered, a further internal control was performed with conventional PCR for the detection of the $\beta$-globin gene. PCR was performed with the primers described by Saiki et al. [10] generating a 268-bp fragment.

In order to investigate the presence of PyV DNA, real-time PCR - SYBR Green and TaqMan methodology - and conventional thermocycler PCR were performed using oligonucleotides complementary to the region called the large T-antigen of each of the PyVs JCPyV, BKPyV, WUPyV, KIPyV, MCPyV, TSPyV, HPyV6, HPyV7, HPyV9, HPyV10, HPyV12, and STLPyV. The protocols used for detection were standardized in house, and the primers were designed using online programs such as Primer3 and PrimerBlast (Rozen and Skaletsky [11] and Ye et al. [12]) (Table 1) [1317]. For the initial screening of $\mathrm{MCPyV}$, a PCR was performed using oligonucleotides complementary to the large $\mathrm{T}$-antigen 
Table 1. List of the primers used in the study

\begin{tabular}{|c|c|c|}
\hline Primer & Fragment size and reference & Sequence \\
\hline PEP1 & $173 \mathrm{pb}$ & AGTCTTTAGGGTCTTCTACC \\
\hline PEP2 & Arthur et al. [16] & GGTGCCAACCTATGGAACAG \\
\hline TSVa_F & $300 \mathrm{pb}$ & CCTGCATGGTGTCAAACAAG \\
\hline TSVa_R & Urbano et al. $[17,22]$ & TGTGCATGGTTTGGAGAAGA \\
\hline MCV LT.1F & $149 \mathrm{pb}$ & CCACAGCCAGAGCTCTTCCT \\
\hline MCV LT.1R & Goh et al. [13] & TGGTGGTCTCCTCTCTGCTACTG \\
\hline WU_1F & $161 \mathrm{pb}$ & TGCACTGTACTAACACACTC \\
\hline WU_1R & New data & CTGGGTCTTGATATGACCTG \\
\hline KI_1F & $255 \mathrm{pb}$ & CCTGGGGGAAATATTTGTGA \\
\hline KI_1R & New data & GTTAAATTTGTGTGGTGGCA \\
\hline V6_F1 & $229 \mathrm{pb}$ & TAACTGTGTAAACCCTCAGC \\
\hline V6_R1 & New data & CAGTTTTTACTGGGGCCTAT \\
\hline V7_F1 & $247 \mathrm{pb}$ & CTACTCTTGCCTTCACTGTT \\
\hline V7_R1 & New data & GAACTGGGATGTGCTATTGA \\
\hline V9_F1 & $214 \mathrm{pb}$ & TAACAGGCCTCCTTCAATTC \\
\hline V9_R1 & New data & GCCTGAACCTAATACTCCAG \\
\hline HPyV10_F & $222 \mathrm{pb}$ & CACCATCAAATTCATGCAAA \\
\hline HPyV10_R & New data & CCAGCAGCAATTCTACCTGT \\
\hline HPyV12_F & $180 \mathrm{pb}$ & CTTTTCCTGGTGAGCCTGTA \\
\hline HPyV12_R & New data & ATCCCTGTGGATCCTTCTTC \\
\hline MWV_F & $150 \mathrm{pb}$ & TCTTTCAATGGATCTTCTCCAG \\
\hline MWV_R & New data & TCTTTGTTGGTGGCAACCTGTA \\
\hline MXV_F & $222 \mathrm{pb}$ & CACCATCAAATTCATGCAAA \\
\hline MXV_R & New data & CCAGCAGCAATTCTACCTGT \\
\hline STLPyV_F & $179 \mathrm{pb}$ & TACCATATTGTCCGCCCTAA \\
\hline STLPyV_R & Urbano et al. [14] & TTGTGTCACTTGGGGAGAAT \\
\hline $\mathrm{PCO} 4$ & $268 \mathrm{pb}$ & CAACTTCATCCACGTTCACC \\
\hline GH20 & Gopalkrishna et al. [15] & GAAGAGCCAAGGACAGGTAC \\
\hline
\end{tabular}

(LTAg) gene described by Pal et al. [18] and both reaction protocols described by Neto et al. [19].

For the detection and genotyping of JCPyV DNA in samples, a fragment from the VP1 gene was amplified by PCR, with the primers JLP-15 and JLP-16 described by Agostini et al. [20]. For BKPyV detection, the same conserved region of the gene encoding the VP1 protein was amplified with the specific BK primers, BKV1 and BKV2, described by Krumbholz et al. [21], confirmed by real-time PCR assay using sets of primers complementary to the other conserved region (LTAg) of each virus (Pal et al. [18]) and parameters followed as described by Urbano et al. [22]. TSPyV DNA detection was performed according to Urbano et al. [17] who used the realtime PCR method for amplification of the conserved region of the LTAg gene.

For the other PyVs, the primers used amplify a conserved region of the LTAg, and the reactions were standardized using by real-time PCR. The mix was prepared using $10 \mu \mathrm{L}$ of the sample (independent of the DNA concentration), $12.5 \mu \mathrm{L}$ of the "SYBR ${ }^{\circledR}$ Green PCR Master Mix" (Applied Biosystems ${ }^{\circledR}$ ), $1.0 \mu \mathrm{L}$ of each primer - final concentration of $0.5 \mathrm{mM}$ - and $5.5 \mu \mathrm{L}$ deionized and distilled water. The parameters of the reaction were $5 \mathrm{~min}$ of $95^{\circ} \mathrm{C}$,

Polyomaviruses in Skin Cancers followed by 40 cycles $\left(30 \mathrm{~s}\right.$ at $95^{\circ} \mathrm{C}$ and $1 \mathrm{~min}$ at the best temperature for each primer and 45 seconds at $60^{\circ} \mathrm{C}$ ) using the $\mathrm{ABI} 7300$ machine (Applied Systems ${ }^{\circledR}$ ) [13]. Thereafter, it was considered positive in those samples presenting an exponential amplification curve during conventional and real-time PCR, with a viral load of $1 \mathrm{e}+03$ copies $/ \mathrm{mL}(900-1.80 \mathrm{e}+05$ copies $/ \mathrm{mL})$, plus a specific $\mathrm{T}_{\mathrm{m}}$ value according to each control sample.

\section{Results}

\section{Samples and Patients}

A total of 140 patients were included in this study. Eleven were excluded because of insufficient material in FFPEM for laboratory analysis or data processing error (6 from group 3 - melanoma and 5 from group 5 - KS). Twenty random samples of normal skin were selected for descriptive purposes of PyV DNA presence, since there 
Table 2. Skin tumours and polyomaviruses

\begin{tabular}{|c|c|c|c|c|}
\hline Skin tumour & $N$ & PyV DNA+, $n(\%)$ & $p$ value & Subtype of PyV DNA ( $n$; \%) \\
\hline BCC & 20 & $3(15)$ & 0.271 & $\begin{array}{l}\text { MCPyV }(2 ; 66.67) \\
\operatorname{SV} 40(1 ; 33.33)\end{array}$ \\
\hline SCC & 20 & $3(15)$ & 0.271 & $\operatorname{SV} 40(3 ; 100)$ \\
\hline Melanoma & 14 & $4(28.57)$ & 0.752 & $\begin{array}{l}\text { MCPyV }(1 ; 25) \\
\text { SV40 }(2 ; 50) \\
\operatorname{STLPyV~}(1 ; 25)\end{array}$ \\
\hline DFSP & 20 & $3(15)$ & 0.271 & $\begin{array}{l}\text { MCPyV }(1 ; 33.33) \\
\text { SV40 }(1 ; 33.33) \\
\text { SV40 + HPyV6 }(1 ; 33.33)\end{array}$ \\
\hline KS & 15 & $2(13.33)$ & 0.346 & $\begin{array}{l}\text { SV40 }(1 ; 50) \\
\text { HPyV6 }(1 ; 50)\end{array}$ \\
\hline MCC & 20 & $13(65)$ & $<0.001$ & $\operatorname{MCPyV}(13 ; 100)$ \\
\hline
\end{tabular}

Correlation between each skin tumour and its control group (composed by samples of all the other tumours). PyV, polyomavirus, BCC, basal cell carcinoma; SCC, squamous cell carcinoma; DFSP, dermatofibrosarcoma protuberans; KS, Kaposi sarcoma; MCC, Merkel cell carcinoma.

Table 3. Immunosuppression and polyomaviruses

\begin{tabular}{llll}
\hline & $\begin{array}{l}\text { Immunosuppressed, } \\
n(\%)\end{array}$ & $\begin{array}{l}\text { Non-immunosuppressed, } \\
n(\%)\end{array}$ & $p$ value \\
\hline PyV DNA-positive samples & $3(16.67)$ & $25(27.47)$ \\
PyV DNA-negative samples & $15(83.33)$ & $66(72.53)$ & 0.555 \\
\hline
\end{tabular}

PyV, polyomavirus.

was no access to their origin data. Therefore, there were 109 patients divided into 20 BCC, 20 SCC, 14 melanoma, 20 DFSP, $15 \mathrm{KS}$, and 20 MCC.

Patients included 50 (45.87\%) women and 59 (54.13\%) men, with a majority of Caucasian patients (89.91\%), followed by Black (7.34\%) and Pardo/brown/mixed ethnic ancestries $(2.75 \%)$. The mean age at the time of diagnosis was 62.7 years ( standard deviation $=19.47$ ). Lesions were predominantly located on the head and neck $(n=35$, $32.11 \%)$ and trunk $(n=35,32.11 \%)$, followed by the upper limbs $(n=17,15.6 \%)$ and lower limbs $(n=14$, $12.84 \%$ ). Other skin cancers were detected in $40.37 \%$ ( $n$ $=44)$, and $16.51 \%(n=18)$ of the patients were immunosuppressed.
Presence of PyV DNA, Groups, and Correlations

PyV DNA was found in $25.69 \%(n=28)$ of the samples: $15 \%$ in BCC $(n=3 / 20), 15 \%$ in SCC $(n=3 / 20), 28.57 \%$ in melanoma $(n=4 / 14), 15 \%$ in DFSP $(n=3 / 20), 13.33 \%$ in KS $(n=2 / 15)$, and $65 \%$ in MCC $(n=13 / 20)$. There was no viral DNA found in group 7 - normal skin.

MCPyV DNA was the most common subtype of PyV detected $(n=17,60.71 \%)$, followed by SV40 $(n=8$, 28.57\%). STLPyV and HPyV6 were each detected only in one sample (3.57\%). In another sample, both SV40 and HPyV6 were detected (3.57\%).

A statistical analysis of the group's results was performed, and the MCPyV DNA detection proportion was statistically significant ( $p$ value $<0.01$ ) in MCC patients (0.65) compared to non-MCC tumour patients 
(0.04-4 MCPyV in 89 non-MCC). There was no statistical correlation between either PyVs and other skin tumours ( $p$ value from 0.27 to 0.75 ) or PyVs and immunosuppression ( $p$ value $=0.513$ ). The control group of each tumour was composed by samples of all the other tumours. A summary of the findings is presented in Tables 2 and 3 .

\section{Discussion}

In recent years, the continuously growing number of newly characterized human PyVs and their potential role in oncogenesis have been described $[23,24]$. In some studies evaluating therapy in advanced MCC, response to antiprogrammed death (PD1) drugs may depend on $\mathrm{MCPyV}$ status, with a higher response rate in $\mathrm{MCPyV}+$ tumours [2]. However, it might be important to evaluate the presence of MCPyV, as well as other PyVs in skin tumours. In addition, the evaluation of immunosuppressed individuals with MCC for MCPyV viral load might be useful.

Several reports have assessed the relationship between MCC and MCPyV with variable results. A meta-analysis study had findings supporting this association: the global proportions of positive samples were 0.79 (95\% CI, $0.72-$ 0.84 ) and 0.12 (95\% CI, 0.08-0.19) in MCCs and control groups, respectively [6]. This association in Brazilian patients showed that $100 \% \mathrm{MCC}$ were positive for MCPyV [19]. Despite our study demonstrating a relatively lower prevalence of positive samples (0.65) in MCC, it is still statistically significant ( $p$ value $<0.01$ ), confirming the important association of MCPyV and MCC. In our work, there was no relationship between any PyVs and other types of skin tumours analysed. In addition, the epidemiological data could not be linked with the prevalence of PyV infection or behaviour of any skin tumours in this specific group of patients. We believe that further studies using prospective analysis might identify epidemiological aspects that could influence the behaviour of these tumours in the light of $\mathrm{PyV}$ infections.

Recent reports had shown the presence of MCPyV DNA in $12-37.5 \%$ of non-MCC skin tumours, with significantly lower viral load than in MCC. MCPyV DNA was markedly positive in non-melanoma skin cancer in immunosuppressed patients as compared to that in nonimmunosuppressed patients $[7,8,19]$. It is believed that HPyVs have the ability to infect the skin and remain in a latent form, which can reactivate in states of profound immunosuppression $[1,2]$.

Polyomaviruses in Skin Cancers
We could not demonstrate the association of PyVs with immunosuppression ( $p$ value $=0.513)$. In contrast, $\mathrm{MCPyV}$ DNA was found in non-MCC tumours such as BCC (10\%), melanoma (7\%), and DFSP (5\%), which developed in non-immunosuppressed individuals, pointing out the presence of MCPyV independent of the immune status in this specific group of patients. Since there is a lack of knowledge about the role of $\mathrm{MCPyV}$ in skin tumours except MCC, further research is needed to demonstrate if HPyVs would influence the behaviour of nonMCC tumours in states of immunosuppression.

There are a few other HPyVs related to skin diseases, such as TSPyV, HPyV6, and HPyV7. TSPyV is one of the etiological agents of trichodysplasia spinulosa, a rare skin disorder characterized by spiny follicular papules and projections. HPyV6 and HPyV7 are also associated with various pruritic and dyskeratotic skin disorders $[25,26]$.

Healthy individuals can chronically shed encapsulated HPyV6 and HPyV7 virions from their skin. HPyV6 has also been detected in actinic keratosis, BCC, SCC, and trichoblastomas, and HPyV6 and HPyV7 have been found in SCC induced by BRAF inhibitors. Immunosuppression increases the risk of developing HPyV6- and HPyV7-associated skin disorders, and significantly higher viral loads are detected in associated skin lesions compared to healthy skin $[2,23,27]$. Besides the considerable number of samples analysed in this study, which included specimens from patients from different regions of Brazil, no PyVs were found in normal skin. Only HPyV6 was identified in 2 cases, one associated with SK and another with DFSP. Since this is the first study to analyse the prevalence of different types of PyVs in the Brazilian population, it is plausible to assume that geographical reasons and ethnic factors might influence this very low prevalence in our country.

STLPyV was first discovered in human faeces. However, a divergent variant was also found in genital warts of a patient with primary immunodeficiency hypogammaglobulinaemia and myelokathexis syndrome, strongly suggesting that it directly infects humans and is not simply a dietary contaminant [4]. We found this virus in a melanoma of a non-immunosuppressed patient, the first report of STLPyV in a skin cancer. Contamination of the sample or a false-positive result is improbable because specific primers were used with rigorous analysis methods. However, the role of this virus in the etiopathogenesis of melanoma is still unknown.

SV40 is able to immortalize human cells and has repeatedly been reported in human tumours such as meso- 
theliomas, brain tumours, and lymphomas, although neither epidemiology nor molecular studies provided convincing evidence for its role in human cancers. It is likely that some of the positive reports in human tumours resulted from a region of homology between LT and cellular DNA, PCR contamination, and the use of vectors containing SV40 sequences or serological cross-reactions with human BK and JC virus antigens [1,9]. Although there are no data about its prevalence in skin tumours so far, we demonstrated a high prevalence of SV40 in tumours such as BCC, SCC, SK, and DSFP. This finding might reflect the influence of geographical and/or ethnic factors; however, future studies are needed to clarify the possible role of SV40 in skin carcinogenesis.

\section{Conclusions}

This study demonstrated the presence of HPyVs in different skin tumours of a specific group of Brazilian patients. It also confirmed the association of $\mathrm{MCPyV}$ with MCC. However, it could not show the relationship of any $\mathrm{PyV}$ identified in different skin tumours with epidemiological data, including immunosuppression. We do believe that further studies are needed to determine the mechanisms of HPyVs in skin carcinogenesis, as well as its interactions with other cofactors such as environmental factors and level of immunosuppression.

\section{Statement of Ethics}

The protocol of this study was approved by the Research Ethics Review Board of Clinics Hospital of the Medical School at the University of São Paulo, as well as its precepts according to the Declaration of Helsinki principles.

\section{Conflict of Interest Statement}

The authors declare that there is no conflict of interest.

\section{Funding Sources}

This work was supported by the authors' own financial resources. This research received no specific grant from any funding agency in the public, commercial, or not-for-profit sectors.

\section{Author Contributions}

Pedro V.A. Costa: conceptualization, data curation, investigation, and writing - original draft, review, and editing; Patricia S. Ishiy: conceptualization, data curation, investigation, and writing - original draft, review, and editing; Paulo R.P. Urbano: conceptualization, data curation, investigation, and writing - original draft; Camila M. Romano: conceptualization, data curation, investigation, supervision, and writing - original draft; Stephen K. Tyring: investigation, supervision, and writing - review and editing; Walmar R.P. Oliveira: conceptualization, data curation, investigation, supervision, and writing original draft, review, and editing; and Cyro Festa-Neto: conceptualization, data curation, investigation, supervision, and writing - original draft, review, and editing.

\section{References}

1 Moens U, Ludvigsen M, Van Ghelue M. Human polyomaviruses in skin diseases. Patholog Res Int. 2011;2011:123491-12.

2 Sheu JC, Tran J, Rady PL, Dao H, Tyring SK, Nguyen HP. Polyomaviruses of the skin: integrating molecular and clinical advances in an emerging class of viruses. Br J Dermatol. 2019: $1-10$.

3 Gheit T, Dutta S, Oliver J, Robitaille A, Hampras $\mathrm{S}$, Combes JD, et al. Isolation and characterization of a novel putative human polyomavirus. Virology. 2017;506:45-54.

4 Pastrana DV, Fitzgerald PC, Phan GQ, Raiji MT, Murphy DH, Velez MD, et al. A divergent variant of the eleventh human polyomavirus species, Saint Louis polyomavirus. Genome Announc. 2013;1(5):2-3.

5 Feng H, Shuda M, Chang Y, Moore PS. Clonal integration of a polyomavirus in human Merkel cell carcinoma. Science. 2008; 319(5866):1096-100.
6 Santos-Juanes J, Fernández-Vega I, Fuentes N, Galache C, Coto-Segura P, Vivanco B, et al. Merkel cell carcinoma and Merkel cell polyomavirus: a systematic review and meta-analysis. Br J Dermatol. 2015;173(1):4249.

7 Dalianis T, Hirsch HH. Human polyomaviruses in disease and cancer. Virology. 2013; 437(2):63-72.

8 Scola N, Wieland U, Silling S, Altmeyer P, Stücker M, Kreuter A. Prevalence of human polyomaviruses in common and rare types of non-Merkel cell carcinoma skin cancer. Br J Dermatol. 2012 Dec;167(6):1315-320.

9 Zur Hausen H. Novel human polyomaviruses: re-emergence of a well known virus family as possible human carcinogens. Int J Cancer. 2008;123(2):247-50.

10 Saiki RK, Gelfand DH, Stoffel S, Scharf SJ, Higuchi R, Horn GT, et al. Primer-directed enzymatic amplification of dna with a thermostable DNA polymerase. Science. 1988; 239(4839):487.
11 Rozen S, Skaletsky H. Primer3 on the WWW for general users and for biologist programmers. Methods Mol Biol. 2000;132:365-86.

12 Ye J, Coulouris G, Zaretskaya I, Cutcutache I, Rozen S, Madden TL. Primer-BLAST: a tool to design target-specific primers for polymerase chain reaction. BMC Bioinformatics. 2012;13(1):134.

13 Goh S, Lindau C, Tiveljung-Lindell A, Allander T. Merkel cell polyomavirus in respiratory tract secretions. Emerging Infect Dis. 2009 Mar;15(3):489-91.

14 Urbano PR, Girardi V, Leal COD, Schneider VE, Paesi SO, Spilki FR, et al. Occurrence of polyomaviruses in recreational freshwaters from Southern Brazil. BJSTR. 2019;14(5):1-5.

15 Gopalkrishna V, Aggarwal N, Malhotra VL, Koranne RV, Mohan VP, Mittal A, et al. Chlamydia trachomatis and human papillomavirus infection in Indian women with sexually transmitted diseases and cervical precancerous and cancerous lesions. Clin Microbiol Infect. $2000 \mathrm{Feb} ; 6(2): 88-93$. 
16 Arthur RR, Dagostin S, Shah KV. Detection of $\mathrm{BK}$ virus and JC virus in urine and brain tissue by the polymerase chain reaction. J Clin Microbiol. 1989 Jun;27(6):1174-179.

17 Urbano PR, Oliveira AC, Romano CM. Alternative methods for sequencing full TSPyV genomes using Sanger or NGS. Rev Inst Med Trop Sao Paulo. 2016 Dec 8;58:92.

18 Pal A, Sirota L, Maudru T, Peden K, Lewis AM Jr. Real-time, quantitative PCR assays for the detection of virus-specific DNA in samples with mixed populations of polyomaviruses. J Virol Methods. 2006;135(1):32-42.

19 Neto CF, Oliveira WRP, Costa PVA, Cardoso MK, Barreto PG, Romano CM, et al. The first observation of the association of Merkel cell polyomavirus and Merkel cell carcinoma in Brazil. Int J Dermatol. 2019 Jun;58(6):703-06.
20 Agostini HT, Deckhut A, Jobes DV, Girones R, Schlunck G, Prost MG, et al. Genotypes of JC virus in East, Central and Southwest Europe. J Gen Virol. 2015;82(Pt 5):1221-331.

21 Krumbholz A, Zell R, Egerer R, Sauerbrei A, Helming A, Gruhn B, et al. Prevalence of BK virus subtype I in Germany. J Med Virol. 2006;78(12):1588-98.

22 Urbano PR, Oliveira RR, Romano CM, Pannuti CS, Fink MC. Occurrence, genotypic characterization, and patterns of shedding of human polyomavirus JCPyV and BKPyV in urine samples of healthy individuals in São Paulo, Brazil. J Med Virol. 2016 Jan;88(1): 153-58.

23 Beckervordersandforth J, Pujari S, Rennspiess D, Speel EJ, Winnepenninckx V, Diaz C, et al. Frequent detection of human polyomavirus 6 in keratoacanthomas. Diagn Pathol. 2016; 11(1):58-60.
24 Decaprio JA, Garcea RL. A cornucopia of human polyomaviruses. Nat Rev Microbiol. 2013;11(4):264-76.

25 van der Meijden E, Janssens RW, Lauber C Bouwes Bavinck JN, Gorbalenya AE, Feltkamp MC. Discovery of a new human polyomavirus associated with Trichodysplasia Spinulosa in an immunocompromized patient. PLoS Pathog. 2010;6(7):e1001024-10.

26 Nguyen KD, Lee EE, Yue Y, Stork J, Pock L, North JP, et al. Human polyomavirus 6 and 7 are associated with pruritic and dyskeratotic dermatoses. J Am Acad Dermatol. 2017; 76(5):932-e3.

27 Schrama D, Groesser L, Ugurel S, Hafner C, Pastrana DV, Buck CB, et al. Presence of human polyomavirus 6 in mutation-specific braf inhibitor-induced epithelial proliferations. JAMA Dermatol. 2014;150(11):1180186 